\title{
Mechanical approaches to oncological drug delivery
}

"The failure to tackle the drug delivery problem may result from the alarming misconception that the poorly perfused hypoxic regions of tumors do not harbor rapidly growing or dangerous target cell populations."

Keywords: cavitation $\approx$ delivery $\approx$ extravasation $\approx$ tumor $\approx$ ultrasound

One of the greatest challenges in oncological drug delivery is achieving successful penetration and distribution of the therapeutic agent throughout the tumor [1].

Several factors combine to make the tumor a challenging environment into which to deliver drugs. The vasculature supporting tumors is characterized by a loss of structure and function with poorly organized, heterogeneous and tortuous vessel architecture. This leads to a 'leaky' vessel phenotype with insufficient pericyte and basement membrane coverage, which in turn causes unregulated extravasation of fluid and solutes into the tumor interstitial space and increased interstitial fluid pressure $[2,3]$. This high-pressure environment causes the collapse of the lymphatic vessels within the tumor which decreases drainage, thereby exacerbating the problem. With an interstitial fluid pressure equivalent to the pressure within the microvasculature $(20 \mathrm{~mm} \mathrm{Hg})$ and dense and disorganized extracelluar matrix, convection from the vascularized regions to the rest of the tumor is very limited [4-6]. With no convective flow through the tumor the movement of molecules becomes entirely dependent on diffusion.

Ultimately the intratumoral deposition of an intravenously delivered cytotoxic drug matches the inefficient, heterogeneous and chaotic nature of the vasculature supplying the tumor. Diffusive passage of therapies against pressure, and through an environment packed with a dense, disorganized 'sieving' extracellular matrix is an extremely inefficient process, a process which becomes even more inefficient with increasing depth into the tumor $[7]$. Although the peak distance between a cell and the nearest blood vessel in healthy tissues rarely exceeds 100 microns, this distance almost doubles to 180 microns in tumors, rendering diffusion-driven delivery even more challenging.
Limited tumoral penetration affects all drug classes including conventional small-molecule chemotherapies and especially larger macromolecular structures. Following intravenous delivery all agents achieve a degree of uptake into the perivascular space next to the blood vessels. Beyond the perivascular space, free drug measuring less than $1 \mathrm{~nm}$ in size will typically exhibit penetration not exceeding $20 \mu \mathrm{m}$ deeper into the tumor, while oncolytic adenovirus measuring $120 \mathrm{~nm}$ does not progress at all. Some novel agents offering the greatest therapeutic promise, such as antibodies, viruses and liposomes are essentially stationary in certain tumor types, while in others they achieve diffusion coefficients as low as $1 \times 10^{-9} \mathrm{~cm}^{2} / \mathrm{s}$ [8]. Liposomal formulations of a typical mean size of $100 \mathrm{~nm}$, such as Doxil ${ }^{\circledR}$, are of particular interest because the enhanced permeability and retention effect allows their increased perivascular accumulation [9]. However, subsequent antitumor activity is hindered by a failure to penetrate and also a failure to release the drug. Indeed, it has been reported that less than a third of the drug reaching tumors is in fact released from Doxil to become bioavailable [10]. For a cancer treatment to be curative, it must have access to all tumor cells, as the survival of just one cell could form the focus of tumor recurrence. Incomplete drug distribution throughout the tumor inevitably leads to incomplete treatment and the grave specter of returning disease.

The failure to tackle the drug delivery problem may result from the alarming misconception that the poorly perfused hypoxic regions of tumors do not harbor rapidly growing or dangerous target cell populations. In fact recent evidence suggests that the exact opposite is true. For example, although gemcitabine treatment diminishes the number of rapidly dividing cells near the blood vessels, a significant proportion of cells in deeper hypoxic regions have been

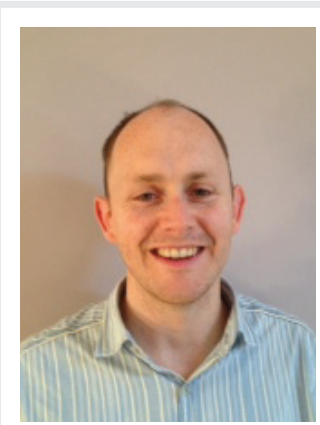

Robert Carlisle

Institute of Biomedical Engineering, Department of Engineering Science, University of Oxford, Old Road Campus Research Building, Oxford OX3 7DQ, UK

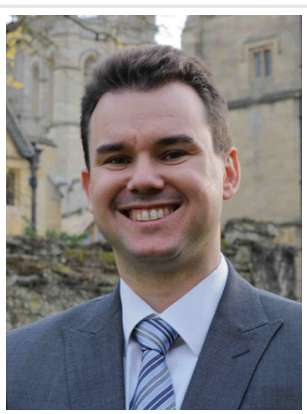

Constantin-C Coussios

Author for correspondence: Institute of Biomedical Engineering, Department of Engineering Science, University of Oxford, Old Road Campus Research Building, Oxford OX3 7DQ, UK

Tel.: +44 1867617727

Fax: +44 1867617728

E-mail: constantin.coussios@

eng.ox.ac.uk 
found to continue to divide unhindered [1]. Furthermore, it is increasingly apparent that these poorly perfused regions may be the sites where cancer stem cells reside and where the process of epithelial-to-mesenchymal transition takes place. These populations and processes have been shown to be associated with an increased likelihood of metastasis and a decrease in 5-year survival [11]. Indeed, those patients with the highest intra-tumoral pressure may be the most likely to suffer recurrence and spread of disease post-treatment [12].

In theory, vascular normalization may offer a pharmacological answer to the problem of poor drug delivery by temporarily restoring normal blood flow through the tumor and providing a window of opportunity during which intratumoral pressure is lowered [3]. However, in practice normalization is not only fleeting but incomplete, widely variable between tumors and difficult to monitor noninvasively [13]. Furthermore, by restoring tumor blood-vessel architecture and function, the enhanced permeability and retention effect, which benefits the tumor accumulation of therapeutic agents such as antibodies, liposomes and viruses, is lost [9]. Indeed, normalization has been shown to actually decrease the tumor accumulation and penetration of liposome-encapsulated doxorubicin [14].

\section{"Today, a new set of mechanical approaches to oncological drug delivery, centered around the use of ultrasound, show great promise as a means of addressing the dual transport problem."}

To date, purely pharmacological approaches have therefore failed to address what is essentially a dual mechanical transport challenge: to actively transport anticancer agents from the vasculature through the leaky endothelium into the perivascular space, and to actively convect the agent against the intratumoral pressure gradient to achieve a therapeutically relevant concentration throughout the tumor.

Today, a new set of mechanical approaches to oncological drug delivery, centered around the use of ultrasound, show great promise as a means of addressing the dual transport problem [15].

Ultrasound is essentially a traveling longitudinal wave of mechanical pressure perturbations, which subjects submillimetric tissue regions to rapidly alternating half-periods of compression and rarefaction of microsecond duration. During the rarefactional half-period, pre-existing gas nuclei can undergo rapid expansion to a size of several microns, followed by a violent collapse during the compressional half-period that is accompanied by the generation of a shockwave. This phenomenon, known as inertial cavitation, generates microstreaming, whereby the bubble imparts momentum onto the surrounding liquid and essentially acts as a micropump that can actively transport molecules away from the bubble surface [16].

In recent mechanistic studies, ultrasoundinduced inertial cavitation has been shown to enable successful delivery of macromolecules over several millimeters against pressure gradients akin to those encountered in tumors [17]. When deployed in the context of one of the most promising yet most challenging therapeutic classes to deliver, oncolytic adenovirus cavitation has also been shown to enable greatly enhanced extravasation and improved distribution in both in vitro and in vivo models [18,19]. In mice bearing human xenograft tumors this resulted in the virus achieving up to 50 -fold increases in tumor infection [18]. Furthermore, the development of several new classes of ultrasound-sensitive delivery vehicles, ranging from liposomes to nanodroplets, make it possible to not only enhance delivery but also achieve targeted release using the same modality $[15,20]$. In addition, the ability to noninvasively detect and map the acoustic phenomena driving therapeutic delivery provides clear potential clinical benefit [21-23].

An exciting common feature of the aforementioned studies is that the levels of ultrasound deployed are akin to those used for diagnostic purposes, potentially presenting a lower regulatory barrier than many other emerging therapeutic modalities. In the context of drug delivery, any enhancements observed are highly likely to be drug-class-agnostic, as the underlying mechanisms are completely mechanical in nature. Ultrasound has been used clinically for over four decades, is widely available, low cost and completely noninvasive. If it can be shown, in clinical studies, to achieve levels of enhanced extravasation and intratumoral distribution akin to those seen in preclinical models, it could provide the long-sought answer to the greatest challenges encountered by oncological drug delivery over the past half century. 
Financial \& competing interests disclosure The authors have no relevant affiliations or financial involvement with any organization or entity with a financial interest in or financial conflict with the subject matter or materials discussed in the manuscript. This includes employment, consultancies, honoraria, stock ownership or options, expert testimony, grants or patents received or pending, or royalties.

No writing assistance was utilized in the production of this manuscript.

\section{References}

1 Minchinton AI, Tannock IF. Drug penetration in solid tumours. Nat. Rev. Cancer 6(8), 583-592 (2006).

2 Boucher Y, Salehi H, Witwer B, Harsh GRT, Jain RK. Interstitial fluid pressure in intracranial tumours in patients and in rodents. Br. J. Cancer 75(6), 829-836 (1997).

3 Jain RK. Normalization of tumor vasculature: an emerging concept in antiangiogenic therapy. Science 307(5706), 58-62 (2005).

4 Baxter LT, Jain RK. Transport of fluid and macromolecules in tumors. I. Role of interstitial pressure and convection. Microvasc. Res. 37(1), 77-104 (1989).

5 Baxter LT, Jain RK. Transport of fluid and macromolecules in tumors. II. Role of heterogeneous perfusion and lymphatics. Microvasc. Res. 40(2), 246-263 (1990).

6 Krol A, Maresca J, Dewhirst MW, Yuan F. Available volume fraction of macromolecules in the extravascular space of a fibrosarcoma: implications for drug delivery. Cancer Res. 59(16), 4136-4141 (1999).

7 Magzoub M, Jin S, Verkman AS. Enhanced macromolecule diffusion deep in tumors after enzymatic digestion of extracellular matrix collagen and its associated proteoglycan decorin. FASEB J. 22(1), 276-284 (2008).

8 Pluen A, Boucher Y, Ramanujan S et al. Role of tumor-host interactions in interstitial diffusion of macromolecules: cranial vs. subcutaneous tumors. Proc. Natl Acad. Sci. USA 98(8), 4628-4633 (2001).

9 Maeda H. The enhanced permeability and retention (EPR) effect in tumor vasculature: the key role of tumor-selective macromolecular drug targeting. Adv. Enzyme Regul. 41, 189-207 (2001).

10 Laginha KM, Verwoert S, Charrois GJ, Allen TM. Determination of doxorubicin levels in whole tumor and tumor nuclei in murine breast cancer tumors. Clin. Cancer Res. 11(19 Pt 1), 6944-6949 (2005).

11 Loges S, Mazzone M, Hohensinner P, Carmeliet P. Silencing or fueling metastasis with VEGF inhibitors: antiangiogenesis revisited. Cancer Cell. 15(3), 167-170 (2009).

12 Milosevic M, Fyles A, Hedley D et al. Interstitial fluid pressure predicts survival in patients with cervix cancer independent of clinical prognostic factors and tumor oxygen measurements. Cancer Res. 61(17), 6400-6405 (2001).

13 Tong RT, Boucher Y, Kozin SV, Winkler F, Hicklin DJ, Jain RK. Vascular normalization by vascular endothelial growth factor receptor 2 blockade induces a pressure gradient across the vasculature and improves drug penetration in tumors. Cancer Res. 64(11), 3731-3736 (2004).

14 Tailor TD, Hanna G, Yarmolenko PS et al. Effect of pazopanib on tumor microenvironment and liposome delivery. Mol. Cancer Ther. 9(6), 1798-1808 (2010).

15 Mo S, Coussios CC, Seymour L, Carlisle R. Ultrasound-enhanced drug delivery for cancer. Expert Opin. Drug Deliv. 9(12), 1525-1538 (2012).

16 Coussios CC, Roy RA. Applications of acoustics and cavitation to noninvasive therapy and drug delivery. Annu. Rev. Fluid Mech. 40, 395-420 (2008).
17 Rifai B, Arvanitis CD, Bazan-Peregrino M, Coussios CC. Cavitation-enhanced delivery of macromolecules into an obstructed vessel. J. Acoust. Soc. Am. 128(5), EL310-EL315 (2010).

18 Bazan-Peregrino M, Rifai B, Carlisle RC et al. Cavitation-enhanced delivery of a replicating oncolytic adenovirus to tumors using focused ultrasound. J. Control. Release 169(1-2), 40-47 (2013).

19 Bazan-Peregrino M, Arvanitis CD, Rifai B, Seymour LW, Coussios CC. Ultrasoundinduced cavitation enhances the delivery and therapeutic efficacy of an oncolytic virus in an in vitro model. J. Control. Release. 157(2), 235-242 (2012).

20 Rapoport N, Gao Z, Kennedy A. Multifunctional nanoparticles for combining ultrasonic tumor imaging and targeted chemotherapy. J. Natl Cancer Inst. 99(14), 1095-1106 (2007).

21 Choi JJ, Coussios CC. Spatiotemporal evolution of cavitation dynamics exhibited by flowing microbubbles during ultrasound exposure. J. Acoust. Soc. Am. 132(5), 3538-3549 (2012).

22 Gyongy M, Coussios CC. Passive cavitation mapping for localization and tracking of bubble dynamics. J. Acoust. Soc. Am. 128(4), EL175-EL180 (2010).

23 Arvanitis CD, Livingstone MS, Vykhodtseva N, McDannold N. Controlled ultrasoundinduced blood-brain barrier disruption using passive acoustic emissions monitoring. PLoS ONE7(9), e45783 (2012). 